\title{
Bi-Luminal Mitral Valve: Incidence, Clinical Features, Associated Anomaly and Echocardiographic Evaluation
}

\author{
Santosh Kumar Sinha, ${ }^{\text {a, }}$, Vikas Mishra ${ }^{\text {a }}$, Karandeep Singh ${ }^{\mathrm{a}}$, Mohammad Asifa, Mohit Sachan, \\ Ashutosh Kumara , Mukesh Jitendra Jha ${ }^{\mathrm{a}}$, Dibbendhu Khanra ${ }^{\mathrm{a}}$, Avinash Kumar Singh ${ }^{\mathrm{a}}$, \\ Shravan Singha ${ }^{\mathrm{a}}$, Mahamdula Razi ${ }^{\mathrm{a}}$, Ramesh Thakur ${ }^{\mathrm{a}}$, Umeshwar Pandeya, \\ Chandra Mohan Varma ${ }^{\text {a }}$
}

\begin{abstract}
Objective: The aim of the study was to know the incidence, clinical features, associated anomaly and echocardiographic evaluation of bi-luminal mitral valve (also known as double orifice mitral valve or DOMV) in patients with suspected mitral valve disease, continous murmur or left-to-right shunt.
\end{abstract}

Methods: Twenty-eight patients with DOMV were diagnosed by transthoracic echocardiography (TTE) in a retrospective review of 52,256 echocardiographic studies in 45,898 patients performed between 2000 and 2015.

Results: The mean age was 20.1 years (15 - 34 years) with female preponderance $(\mathrm{M} / \mathrm{F}$ : 1:1.8). Dyspnea and diastolic murmur were the most common symptoms found in $19(67.8 \%)$ and $19(67.8 \%)$ of patients, respectively. Normal sinus rhythm was the most common electrocardiographic finding. Twenty-five (89\%) patients had complete bridge, while three $(11 \%)$ had incomplete bridge type of DOMV. Twenty-one (75\%) had severe mitral stenosis (MS) including severe tricuspid regurgitation $(\mathrm{n}=13,61 \%)$, ventricular septal defect (VSD, $\mathrm{n}=3,14 \%)$, complete endocardial cushion defect (ECD, $\mathrm{n}=3,14 \%)$, and mild to moderate mitral regurgitation $(\mathrm{MR})(\mathrm{n}=2,11 \%)$, moderate MS and moderate MR were found in four $(16 \%)$ patients among complete bridge type of DOMV, while all patients with incomplete bridge type had severe MS and patent ductus arteriosus (PDA) as associated lesions. Overall, 24 (85\%) had severe and four (15\%) had moderate MS.

Conclusions: DOMV as a cause of symptomatic mitral valve disease was seen in young and middle-aged patients with estimated incidence of $0.06 \%$. Dyspnea and diastolic murmur were the most common symptoms. Mostly, it was an isolated anomaly but in majority, associated with VSD, complete ECD and PDA. TTE examination is a

Manuscript accepted for publication October 10, 2016

aDepartment of Cardiology, LPS Institute of Cardiology, G. S. V. M. Medical College, Kanpur, Uttar Pradesh 208002, India

${ }^{b}$ Corresponding Author: Santosh Kumar Sinha, Department of Cardiology, LPS Institute of Cardiology, G. S. V. M. Medical College, Kanpur, Uttar Pradesh 208002, India. Email: fionasan@rediffmail.com

doi: http://dx.doi.org/10.14740/jocmr2790w reliable and sufficient means of diagnosing DOMV and determining its type.

Keywords: Complete endocardial cushion defect; Double orifice mitral valve; Patent ductus arteriosus; Primum atrial septal defect; Ventricular septal defect

\section{Introduction}

Double orifice mitral valve (DOMV) is an exceedingly rare congenital malformation occurring in $0.05 \%$ of the general population [1]. It was first described by Greenfield in 1876 [2]. DOMV is characterized by two valve orifices in the left atrio-ventricular (AV) valve area with two separate subvalvular apparatus [3, 4]. It is thought to result from abnormal fusion of the endocardial cushions and abnormal development of the mitral valve from the primary fold and primitive left ventricle during a delamination process [5]. According to Lewis, which seems more convincing, the double mitral valve is the result of an early arrest of development and the accessory orifice represents a retention of the left portion of the common AV canal with subsequent reduction of the mitral ostium and alignment with it [6]. A DOMV may occur as an isolated anomaly, but in most of cases, it is associated with other congenital malformations. The hemodynamic impact of DOMV varies from a normally functioning valve to (very rare), congenital mitral stenosis (MS) or significant mitral regurgitation (MR) (with or without cleft) [7]. The purpose of our study was to find the incidence, clinical presentation, associated anomaly and detail echocardiographic study of DOMV evaluated with initial diagnosis of mitral valve disease or congenital heart disease with left-to-right shunt.

\section{Materials and Methods}

A retrospective review was carried out on patients with DOMV diagnosed between 2000 and 2015 in our echocardiographic station among patients being referred for echocardiographic evaluation of murmur, with an initial diagnosis of either mitral valve disease or congenital heart disease with left-to-right shunt. Detailed clinical examination record in- 
cluding electrocardiogram was reviewed. Two-dimensional (2D) transthoracic echocardiographic (TTE) study was performed in all patients by GE-Vivid 6 and $7^{\mathrm{TM}}$ ultrasound system after proper consent. In addition, few patients had undergone 3D TTE study as well, performed by same machine and transesophageal echocardiographic (TEE) study if required. 2D images in standard view, i.e., parasternal longand short-axis views (PLAX and PSAX) and apical four- and two-chamber views (A4C and A2C), were acquired for the delineation of DOMV and its associated lesions. DOMV was diagnosed on the basis of valve morphology visualized in different views as proposed by Trowitz et al [7]. According to this, this anomaly has three different types: complete bridge, incomplete bridge and hole type. The complete bridge type (about $15 \%$ of DOMVs) is characterized by one in which both orifices are visible from the leaflet edge, all the way through the valve ring. Both openings are circular (equal or unequal in size), and papillary muscles usually are normal, with chordae surrounding each orifice inserting into one papillary muscle. The incomplete bridge type is one in which the connection is seen only at the leaflet edge, resulting in a double circle only at the leaflet level, with a normal appearance in the more basal views. The hole type, the most common variety, is characterized by a small accessory orifice situated at either the anterolateral or posteromedial commissural, visible only at the mid-leaflet level and disappearing on scanning toward the apex or base. When the accessory orifice is located at the posteromedial commissure, a common AV canal usually is present $[3,7,8]$. The echocardiographic study was completed by comprehensive Doppler imaging (evaluation of the diastolic pressure gradient across the mitral valve, the mitral valve area and the degree of MR). TEE had been preformed after proper consent with conscious sedation by xylocaine spray after ruling out contraindications in left lateral position and images were again acquired in standard view.

\section{Results}

\section{Baseline and clinical characteristics}

Overall, 28 patients with DOMV were identified among 52,256 echocardiographic studies in 45,898 patients performed between 2000 and 2015. The mean age was 20.1 years (range: 5 - 41 years). There was a female preponderance with sex ratio of $1: 1.18$ (female $=18$; male $=10$ ). The clinical symptoms of patients were dyspnea $(n=19,67.8 \%)$, palpitation $(n=6,21.4 \%)$; fatigue $(n=4,14.2 \%)$, hemoptysis $(\mathrm{n}=2,7.1 \%)$ and embolic phenomenon $(\mathrm{n}=2,7.1 \%)$ (Table 1). The one with embolic phenomenon presented with severe pain in both lower limbs with bilateral loss of pulse. Saddle embolus at common iliac bifurcation was recorded and urgent embolectomy had been performed. Their functional class was New York Heart Association (NYHA) I, II, III and IV in seven $(25 \%), 17(60.7 \%)$, five $(17.9 \%)$ and $0(0 \%)$, respectively. On cardiovascular symptom examination, diastolic thrill, systolic thrill and continous thrill were palpatory findings in $14.2 \%$, $14.2 \%$ and $10 \%$, respectively. S1 was loud in most of them
Table 1. Baseline Characteristics and Clinical Presentation of Patients $(n=28)$

\begin{tabular}{|ll}
\hline Variables & No. $(\%)$ \\
\hline Age (mean \pm SD) & $20.1 \pm 4.9$ \\
Gender (male/female) & $10 / 18(1: 1.8)$ \\
Clinical presentation & \\
$\quad$ Dyspnea & $19(67.8)$ \\
$\quad$ Palpitation & $6(21.4)$ \\
Fatigue & $4(14.2)$ \\
\hline Hemoptysis & $2(7.1)$ \\
Embolic phenomenon & $2(7.1)$ \\
NYHA functional class & \\
\hline I & $7(25)$ \\
II & $17(60.7)$ \\
III & $4(14.3)$ \\
IV & - \\
\hline CVS examination & \\
\hline Diastolic thrill & $4(14.3)$ \\
Systolic thrill & $4(14.3)$ \\
Continuous thrill & $3(10.7)$ \\
Diastolic murmur & $19(67.8)$ \\
Pansystolic murmur & $12(42.8)$ \\
Continuous murmur & $3(10.7)$ \\
ECG & $25(89)$ \\
Normal sinus rhythm & $3(10.7)$ \\
Atrial flutter/fibrillation & $3(10)$ \\
\hline
\end{tabular}

CVS: cardiovascular system; ECG: electrocardiogram; LAD: left axis deviation; NYHA: New York Heart Association.

and variable in few. S2 was loud with narrow split. Long diastolic, pansystolic and continous murmur were auscultator findings in $19(67.8 \%), 12(42.8 \%)$ and three $(10.5 \%)$, respectively. On electrocardiogram, 25 (89\%) had normal sinus rhythm, while atria flutter/fibrillation was identified in three $(10.7 \%)$ and left axis deviation in three $(10.7 \%)$ of patients, respectively (Table 1).

\section{Echocardiographic evaluation and associated lesion}

In 25 patients $(89 \%)$, the complete bridge type of DOMV was recognized (Table 2). Of them, severe MS was identified in 21 $(84 \%)$ of patients with average valve area of $0.4-0.8 \mathrm{~cm}^{2}$ by planimetry (Fig. 1C), and pressure half time (PHT) (Fig. 1D). Their mean gradient was $14-28 \mathrm{~mm} \mathrm{Hg}$. Those with severe MS, isolated MS with secondary tricuspid regurgitation (TR) were found in 13 (47\%) (Fig. 1A), mild to moderate MR in two $(7.1 \%)$, ventricular septal defect with left-to-right shunt in three $(11 \%)$, and complete endocardial defect in three $(11 \%)$ 
Table 2. Details of Echocardiographic Evaluation of Patients With DOMV $(n=28)$

\begin{tabular}{|c|c|c|c|c|c|}
\hline \multirow{2}{*}{ Type of DOMV } & \multicolumn{3}{|c|}{ Mitral stenosis } & \multirow{2}{*}{ Associated lesions } & \multirow{2}{*}{$\mathbf{N}(\%)$} \\
\hline & Severity & $\operatorname{MVA}\left(\mathrm{cm}^{2}\right)$ & Gradient (mean mm Hg) & & \\
\hline \multirow[t]{3}{*}{ Complete bridge type ( $89 \%)$} & \multirow[t]{3}{*}{ Severe } & \multirow[t]{3}{*}{$0.4-0.8$} & \multirow[t]{3}{*}{$14-28$} & Severe TR & $13(46)$ \\
\hline & & & & Mild to moderate MR & $2(7)$ \\
\hline & & & & ECD (complete) - primum ASD and VSD & $3(11)$ \\
\hline
\end{tabular}

DOMV: double orifice mitral valve; MVA: mitral valve area; TR: tricuspid regurgitation; VSD: ventricular septal defect; MR: mitral regurgitation; ECD: endocardial cushion defect; ASD: atrial septal defect; $P D A$ : patent ductus arteriosus; $V_{\max }$ : maximum peak velocity; $P G_{\text {max }}$ : maximum peak gradient.

of patients (Fig. 2B, C, D). Those with MR were better recognized on TEE. Two patients had multiple ventricular septal defects among those with complete endocardial cushion defect (ECD) (Fig. 2B). Moderate MS with moderate MR (Fig. 1B) was identified in four (14\%) of patients. TR was present either in isolated type of DOMV (secondary) or DOMV associated with complete ECD but was absent in DOMV associated with patent ductus arteriosus (PDA).

Incomplete bridge type of DOMV was identified in three $(10 \%)$ of patients. All had severe MS and PDA with left-toright shunt was accompanying lesion in all of them (Fig. 3). The 2D TTE was sufficient to define the type, anatomy and associated lesions in all cases though 3D TTE was also done in one case with PDA for better delineation. TEE was needed for better quantification of degree of MR in one patient as TTE was equivocal and revealed mild MR.

\section{Discussion}

The normal mitral valve consists of a central orifice located between a sail-like anterior leaflet and a C-shaped posterior leaflet. The anterior mitral leaflet occupies roughly one-third of the annular circumference and is wider than the posterior leaflet. The posterior leaflet occupies roughly the remaining two-thirds of the annular circumference and is narrower. Although morphologically different, the surface areas of the anterior and posterior mitral valve leaflets are nearly the same. In a DOMV, however, abnormal tissue divides the mitral orifice into two parts because of developmental arrest.
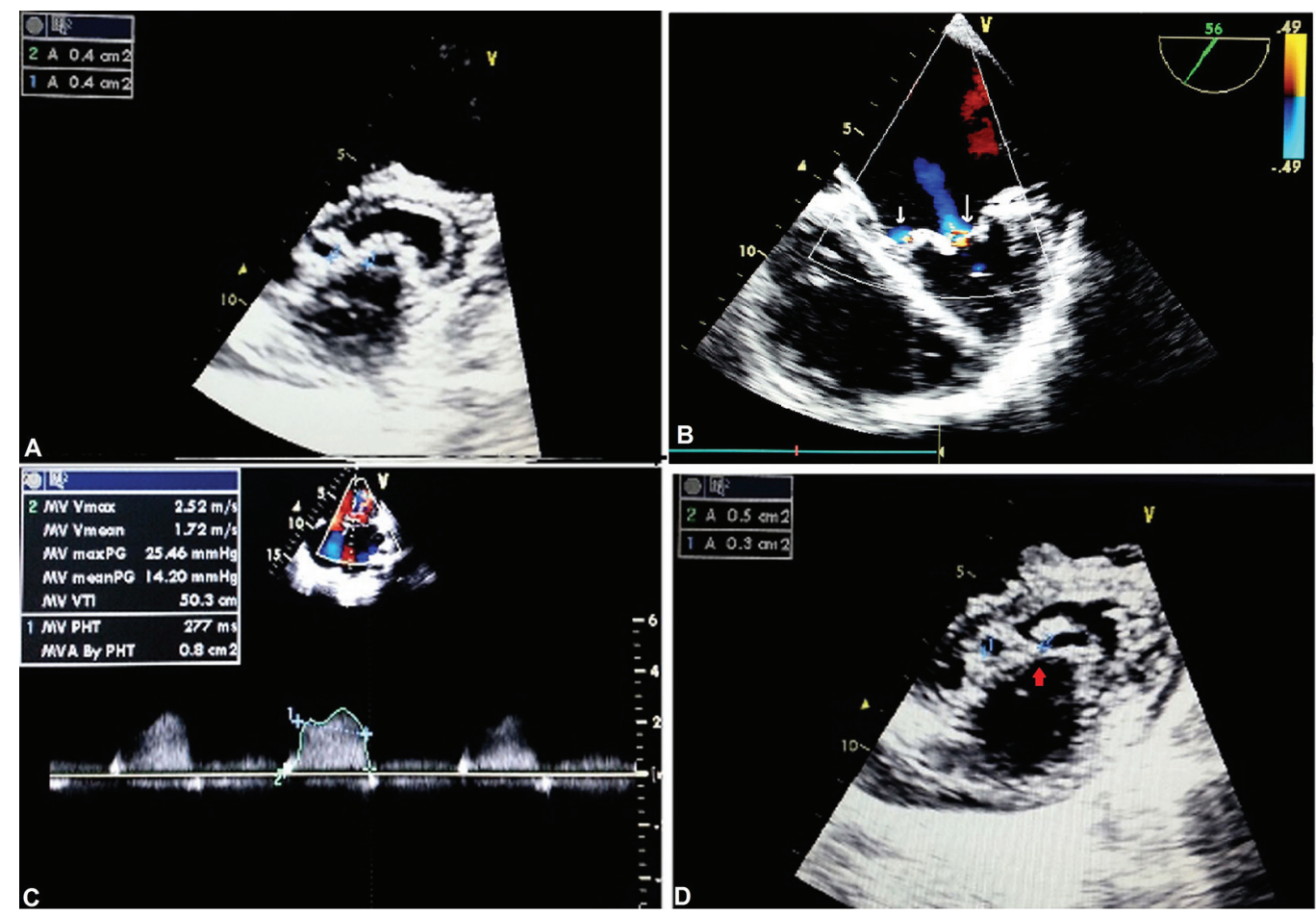

Figure 1. Complete bridge type of DOMV: apical four-chamber view showing TR (A); TEE showing mitral regurgitation, parasternal (B); short-axis (PSAX) view showing severe mitral stenosis by pressure half time (C) and planimetry (D). 


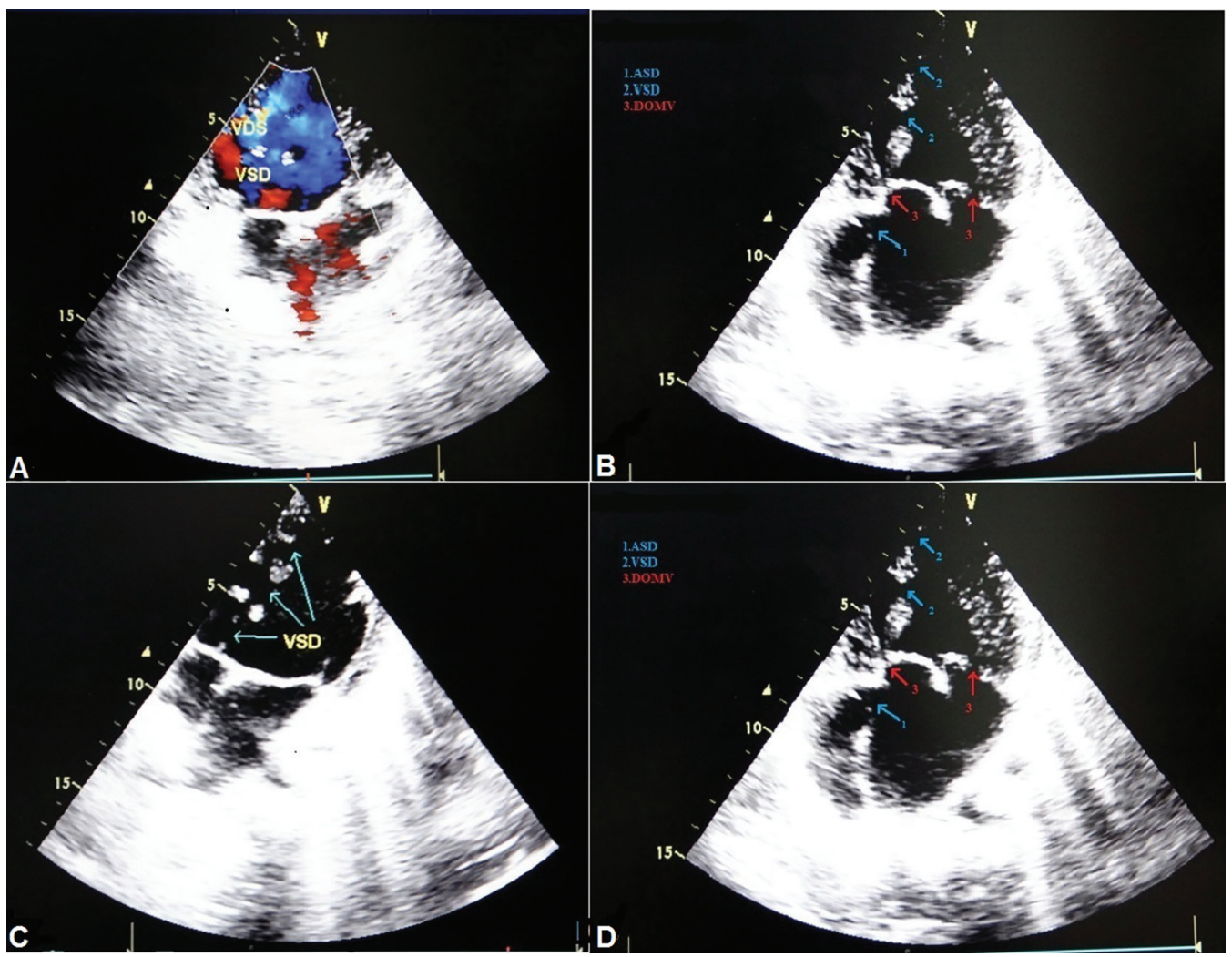

Figure 2. DOMV with ventricular septal defect with left-to-right shunt (A, B) and complete endocardial cushion defect (C, D).

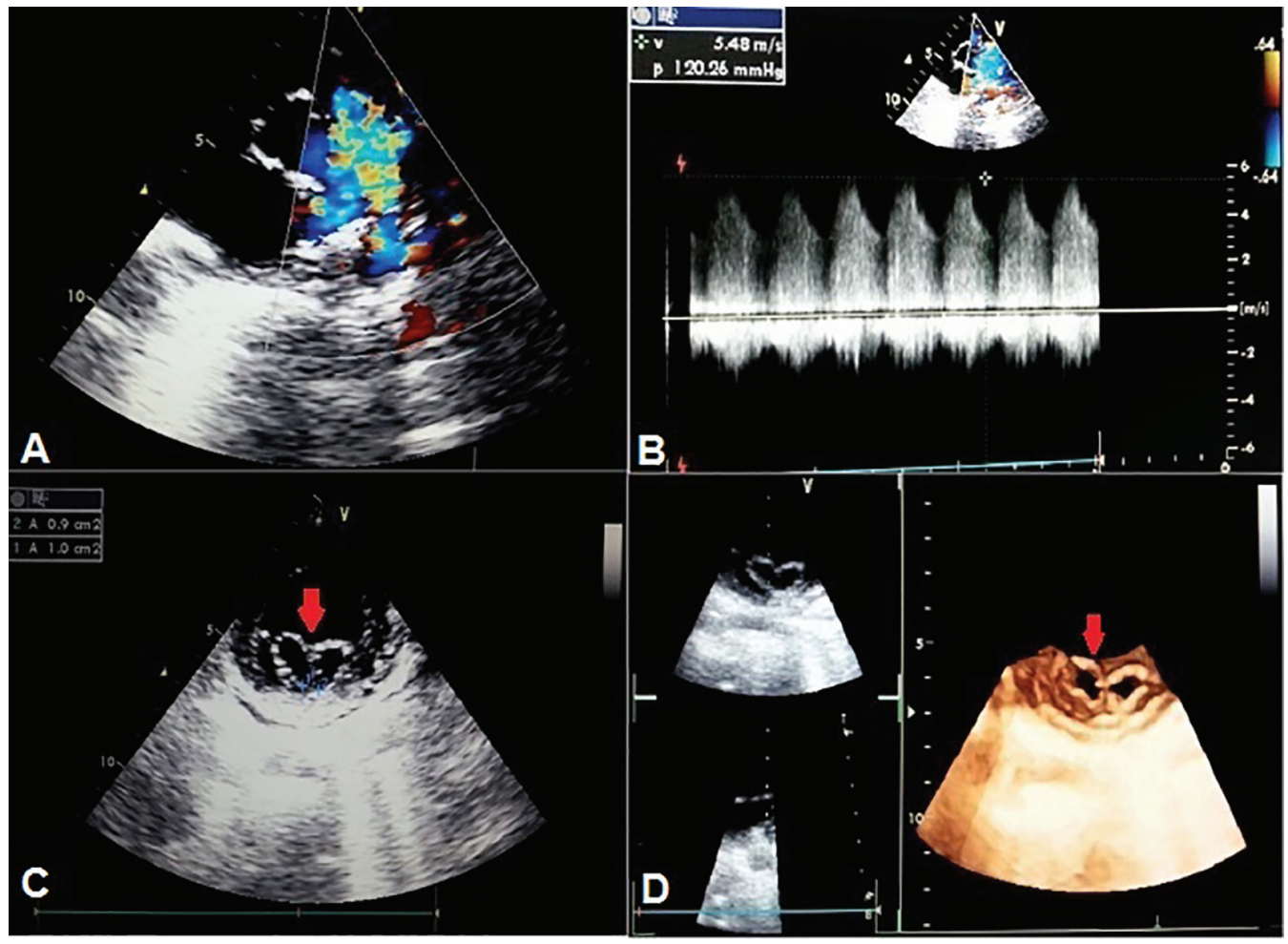

Figure 3. TTE basal short-axis view showing patent ductus arteriosus $(A, B)$. Incomplete bridge type of DOMV on 2D (C) and 3D transthoracic echo (D). 
The clinical presentation of DOMV spans from asymptomatic to varying degree of regurgitation or stenosis. It is primarily governed by the associated lesions and the functional status of both orifices $[1,9]$. Although DOMV may allow normal hemodynamic flow between the left atrium and left ventricle, it may substantially obstruct mitral valve inflow causing either stenosis or mitral valve incompetence because of improper cooptation. Function of both components of the DOMV is mildly impaired in most of cases but at times can be normal in some patients. Mild to severe regurgitation or significant stenosis of either or both orifices has been reported [10]. The DOMV can be an isolated anomaly, but in most cases, it is associated with other congenital malformations which may be either cyanotic or cyanotic such as AV canal defect, ventricular septal defect, left ventricular outflow tract (LVOT) obstruction, coarctation of the aorta, interrupted aortic arch, patent arterial duct, primum type atrial septal defect (ASD), tetralogy of Fallot or Ebstein's anomaly [5]. MR is the most common functional abnormality, and stenosis, either alone or with insufficiency, is uncommon [5, 7]. In our group, severe MS and mild MR were the predominant findings. It was associated with both the complete bridge and incomplete bridge types of DOMV. Bridge type was the only variety noted in our group. Though hole type of DOMV is thought to be the most common type (more than $80 \%$ of patients) and is often associated with a common AV canal [8], in our group, the hole type was diagnosed in none and the one having complete ECD was having complete bridge type of DOMV. In one of the largest groups of 46 children with DOMV described by Zalzstein et al [3], MR was the most frequent finding (43\%), whereas MS was present in $13 \%$ of cases, and combined MS and insufficiency in $6.5 \%$ of patients. The most commonly associated anomaly was AV septal defect $(39 \%)$ but in our study, it was only $16 \%$. Severe stenosis was the most common finding in our group. There are no specific clinical signs suggestive of DOMV [5]. Most of the referrals were for evaluation of dyspnea where mitral valve disease was suspected. All those with PDA were referred for evaluation of continuous murmur. One who had ECD was being evaluated for dyspnea and left axis deviation in electrocardiogram .

Though DOMV is an exceedingly uncommon anomaly, one should be aware of its presence when examining patients with mitral valve disease even in Indian subcontinent where mitral valve affliction means rheumatic etiology. The hallmark of rheumatic involvement is leaflet thickening $(>5 \mathrm{~mm})$, fixed posterior mitral leaflet, commissural fusion and varying degree of subvalvular apparatus involvement which were characteristically absent in DOMV [11]. According to Bano-Rodrigo et al [6], the frequency of equal-sized orifices in patients with DOMV is limited to $15 \%$, with the remainder being unequal in size. Our findings did match the same. Of particular interest was that all had predominant stenosis and most of them were of complete bridge type. In our retrospective review of 52,256 echocardiographic studies of 45,898 patients performed between 2000 and 2015, only six patients of DOMV were identified with incidence of $0.06 \%$. In $11 \%$ of patients, complete ECD and PDA were found. This is a much lower rate than that recorded at the Children's Hospital, Denver, USA, where 18 patients with an intact AV septum were identified from 40,179 echocardiographic studies performed between 1997 and 2002
[4]. This however can be easily explained by the different clinical profiles of a pediatric and an adult echocardiographic laboratory and also the fact that rheumatic etiology predominates in our country. Because there are no specific clinical signs to suggest the diagnosis of a DOMV, echocardiography is the method by which this anomaly is detected. Treatment may be either surgical repair or replacement. Replacement is preferable for fibrocalcific valves, MS with insufficiency, and where repair is not feasible.

\section{Conclusions}

DOMV as a cause of symptomatic mitral valve disease is also seen in middle-aged/elderly. Most of the patients stay in normal sinus rhythm. TTE examination, especially in short-axis parasternal views, is a reliable method and in most of cases, sufficient to confirm a diagnosis of DOMV and to determine its type. The incidence of the diagnosis of DOMV in the adult tertiary referral echocardiographic laboratory is $0.06 \%$.

\section{Conflicts of Interest}

None.

\section{Funding Sources}

None.

\section{References}

1. Banerjee A, Kohl T, Silverman NH. Echocardiographic evaluation of congenital mitral valve anomalies in children. Am J Cardiol. 1995;76(17):1284-1291.

2. Greenfield WS. Double mitral valve. Transactions of the Pathological Society of London. 1876;27:128-129.

3. Zalzstein E, Hamilton R, Zucker N, Levitas A, Gross GJ. Presentation, natural history, and outcome in children and adolescents with double orifice mitral valve. Am J Cardiol. 2004;93(8):1067-1069.

4. Das BB, Pauliks LB, Knudson OA, Kirby S, Chan KC, Valdes-Cruz L, Cayre RO. Double-orifice mitral valve with intact atrioventricular septum: an echocardiographic study with anatomic and functional considerations. J Am Soc Echocardiogr. 2005;18(3):231-236.

5. Erdemli O, Ayik I, Karadeniz U, Yamak B, Birincioglu CL, Caglar K. A double-orifice atrioventricular valve case: intraoperative transesophageal echocardiography in diagnosis and treatment. Anesth Analg. 2003;97(3):650653.

6. Bano-Rodrigo A, Van Praagh S, Trowitzsch E, Van Praagh R. Double-orifice mitral valve: a study of 27 postmortem cases with developmental, diagnostic and surgical considerations. Am J Cardiol. 1988;61(1):152-160.

7. Trowitzsch E, Bano-Rodrigo A, Burger BM, Colan SD, 
Sanders SP. Two-dimensional echocardiographic findings in double orifice mitral valve. J Am Coll Cardiol. 1985;6(2):383-387.

8. Wojcik A, Klisiewicz A, Lusawa T, Hoffman P. [Double-orifice mitral valve - case report]. Kardiol Pol. 2005;63(6):663-665.

9. Jose VJ, Chandy ST, John B. Cardiovascular images. Double-orifice mitral valve. Indian Heart J. 2003;55(3):279-
280.

10. Hoffman P, Stumper O, Groundstroem K, Sutherland G. The transesophageal echocardiographic features of double-orifice left atrioventricular valve. J Am Soc Echocardiogr. 1993;6(1):94-100.

11. Lo PH, Hung JS, Lau KW, Kim MH, Ku PM, Krayyem M. Inoue-balloon mitral valvuloplasty in double-orifice mitral stenosis. J Invasive Cardiol. 2003;15(6):301-303. 